\title{
An Automatic Grading System for Panels Surfaces Using Artificial Vision
}

\author{
Cristhian Aguilera, Mario Ramos, Gabriel Roa
}

\begin{abstract}
This work describes an automatic grading system using artificial vision to improve the quality of wood panels surfaces. The objective is to control stains on the surface. Artificial Vision techniques like Thresholding and Transformed Watershed methods are applied. Defects quantitative measures found on the surface are also presented, in particular quantity, area, intensity and distribution.

Keywords:Vision, Image Processing, Quality Control, Plywood
\end{abstract}

\section{Introduction}

The need of manufacturing companies to maintain high quality product requires an exhaustive production control during and at the end of the process. When control is human visual inspection product control is not completely reliable and it is not guaranty of a total quality control.

However, the development of new technologies, and especially image analysis systems (Artificial Vision), has ostensibly improved the quality control process. Since it is a noninvasive technology and is capable to inspect the $100 \%$ of the production, Artificial Vision offers an advantage when compared with the manufactured product quality control techniques like human inspection and Sampling.

The wood industry and specifically in the panels industry has produced efforts to improve each, every one of the production stages. Two main characteristics determine the panel quality: mechanical resistance and appearance. Artificial Vision (Garrido, 2003) is a solution for the appearance control quality. Panels like plywood are used in the carpentry industry. Decorative paneling is the reason why surface quality must be controlled. Since the objective of these products is both functional and esthetic.

The main objective of this paper is to propose an analytical Artificial Vision based method to classify panels according to the Quantity, Area, Intensity, and Stains Distribution on the surface. It includes among these characteristics quantitative methods to measure the stain distribution on the panels. Factors to permit the quantification of these parameters and especially of the stain distribution in this paper are proposed.

\section{Panels}

\subsection{Definition}

Panels can be defined (Corma, 2003).

Structural Panels this term refers to those panels employed as structural elements in the construction and packing industry, included plywood.

Non-structural panels this term refers to those panels employed in the furniture industry, included decorative plywood, hard panels, and Medium Density Fiberboard, MDF.

\subsection{Classification}

Traditionally, wood, and consequently wood-derived products, has been classified considering two fundamental criteria: resistance and appearance.

In relation to the resistance, the objective is to control the mechanical resistance degree. There are standards that these products should satisfy. Tests are related with the mechanical quality evaluation like thickness, density, weight, flexion, traction, elasticity, and humidity. 
In the case of appearance, the objective is the visual appearance control, considering esthetic more than structural values. These criteria are important in the furniture and paneling industry. Defects affecting the surface quality, especially in MDF and fiber panels, are the stains on the surface.

As an example, a summary of the characteristics considered by a Chilean company to grade surface quality of MDF and fiber panels are presented in Table 1. It can be observed that panel grading is divided in three types.

- First quality (I).

- Second quality (II).

- Third Quality (III).

\section{Industrial vision system}

Industrial Vision Systems are rapidly becoming a key factor in the development of total quality procedures in an industrial automation processes context. Industrial Vision Systems permit to inspect production processes without fatigue or distraction, facilitating the quantification of quality variables and contributing to a continuous improvement (Silvén and Matti, 2003). The figure 1 presents the outline of the stages of an Industrial Vision System.

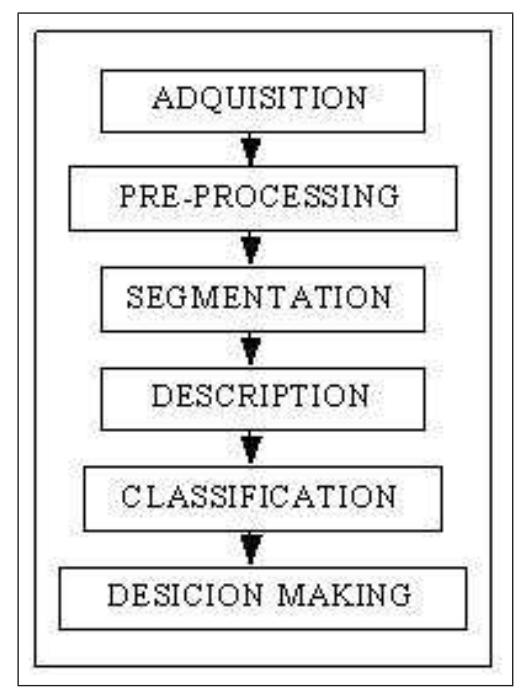

Figure 1: Industrial Vision System stages

\subsection{Image Acquisitions and Digitalization}

This is the stage in which sensors and the capacity to digitalize the signal produced by the sensor are used. The sensor is either color or monochrome camera that produces a complete image. After capturing the image, this information is sent to the computer to be analyzed (Mery, 2003).

\subsection{Pre-processing}

In this process, the acquired image is modified in order to improve it according to the parameters to analyze considering:

- Noise elimination. 


\begin{tabular}{|c|c|c|}
\hline & MDF & FIBER \\
\hline QUAL & Surface & Surface \\
\hline $\mathrm{I}$ & $\begin{array}{l}\text { Both faces free of porosity, } \\
\text { cracks, semi-inflated and dark } \\
\text { stains }\end{array}$ & $\begin{array}{l}\text { Both faces free of porosity, cracks, un- } \\
\text { sanded holes, semi-inflated, and stains } \\
\text { falling from the surface }\end{array}$ \\
\hline I & $\begin{array}{l}\text { Minor stains are permitted when } \\
\text { there are no more than } 2 \text { per face } \\
\text { and they are smaller than } 2 \mathrm{~cm}\end{array}$ & $\begin{array}{l}\text { Longer strips that do not surpass } 0.5 \\
\mathrm{~cm}^{2} \text { in area are permitted. The quan- } \\
\text { tity should be less than } 10 \text { per face and } \\
\text { presenting a separation greater than } 5 \\
\text { cms. between them }\end{array}$ \\
\hline I & $\begin{array}{l}\text { Good behavior during milling } \\
\text { and coating application. }\end{array}$ & $\begin{array}{l}\text { Minor stains are permitted if they are } \\
\text { not greater than } 5 \mathrm{~cm}^{2} \text { of area. The } \\
\text { quantity should be less than } 5 \text { per face } \\
\text { and with a separation greater than } 5 \mathrm{~cm}\end{array}$ \\
\hline II & $\begin{array}{l}\text { Cracks, thick particles, dust, ad- } \\
\text { hesive, or other agent stains, and } \\
\text { semi-inflated }\end{array}$ & $\begin{array}{l}\text { Porosity, cracks, un-sanded holes, thick } \\
\text { particles on the surface, adhesive, dust, } \\
\text { or other stains }\end{array}$ \\
\hline III & Without quality requirements & Without quality requirements \\
\hline
\end{tabular}

Table 1: Characteristics for panel grading according to the surface defects

- Accentuate or profile image characteristics (borders, limits, etc).

- Improve the quality of some parts of the image.

\subsection{Segmentation}

The segmentation process divides the digital image in unconnected regions in order to separate region of interest from the rest of the scene. In the last years, diverse segmentation techniques have been developed, which can be grouped in three techniques: pixel-oriented, border-oriented, and region-oriented techniques.

\subsection{Description}

This process labels the objects considering information supplied by inspection that can be:

Quantitative: Measuring of areas, lengths, perimeters, etc.

Qualitative: Verification of correct task performance (assembling, bottling, labeling, etc.).

\subsection{Classification}

Classification orders the segmented regions in classes, assigning to each region a group of many preestablished groups that represent all the possible types of regions that are expected to exist in the image. In this stage, a statistical study is performed on the characteristics that are extracted from the objects whose defects are known a priori.

\subsection{Decision Making}

Frequently, computer vision systems control a mechanical apparatus that manipulate the products after classification. 


\section{Proposed techniques}

To grade the panel surface defects, image analysis techniques need to be used. This stage differentiates one vision system from another. The analysis is performed on images using a gray scale where the intensity values for black are 0 and 255 for white. One of the techniques to be used is thresholding (Guindos, 2001). Panel surface stains are considered regions. Another segmentation technique is the one based in growth of the regions applying functions under morphological operators, specifically the Transformed Watershed.

\subsection{Thresholding}

The thresholding technique (Gonzales and Goods, 1996) classifies each pixel in two groups, depending on whether or not the gray level exceeds the given threshold. If it does not exceed, then a lower gray level (color black) is assigned; in the opposite, the upper level (white color) is assigned. The objective is to obtain an image segmentation, creating black stains on a white background in a form that is similar to the objects seen from an upper plane, as can be observed in Figure 2.

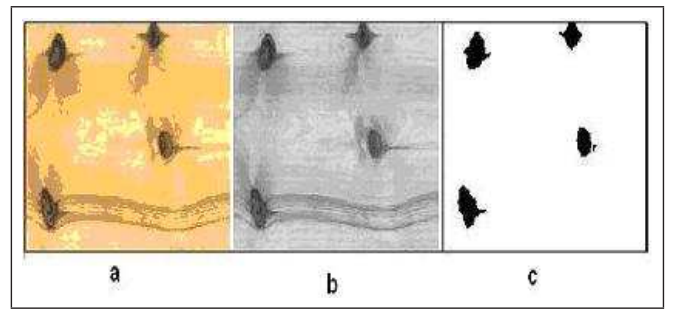

Figure 2: (a) original image, (b) I. Gray-scaled image (c) Thresholded Image

\subsection{Transformed Watershed}

This technique is part of a series of algorithms emerging to analyze images as a topographic surface where height is equal to the intensity. The highest pixel values correspond to the highest land areas and vice versa.

The Transformed Watershed simulates, based in the minimum values of the image of water filling the image, figure 3. Also, these points can be randomly selected, called Markers. The point where the water comes from two regions is called the Watershed and is the point that separates the basins corresponding to two minimums.

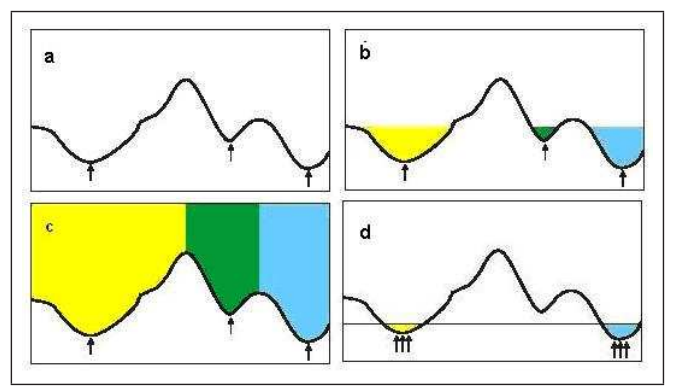

Figure 3: (a) Marker selection, (b) Flooding process from the markers, (c) Finalized Segmentation, (d) Selection of markers using thresholding

The key to segment using the Transformed Watershed is the markers selection. If each local minimum is taken, it could produce an over-segmentation. In order to reduce over-segmentation, the original image 
is frequently softened before performing segmentation.

With the thresholding method, a set of markers can be obtained in which a pre-determined filling level is fixed. Figure 4 is an example of the over-segmentation when the Transformed Watershed is applied to the original image without establishing local minimums.

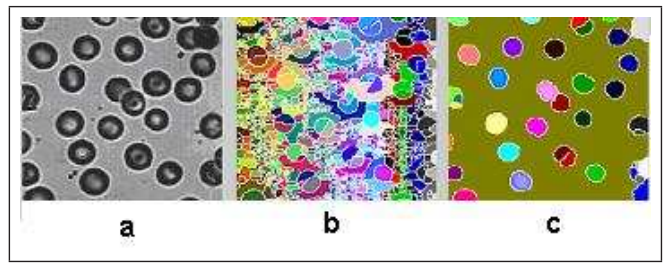

Figure 4: Watershed Results: (a) original image, (b) over-segmented image, (c) segmented image using local minimums

\section{Extraction of panel characteristics}

When the stains (defects) are found numeric properties are extracted. These characteristics provide information to grade the panel. The characteristic are Quantity, Area, Intensity and Distribution.

\subsection{Quantity}

This characteristic indicates the number of stains on the panel surface and whose factor is defined by the quantity of stains.

$$
F C=C_{T}
$$

where: $F C$ is the quantity factor, $C_{T}$ is the number of stains present on the panels surface.

\subsection{Area}

This characteristic indicates the stained surface of the panel. Its value is defined by the ratio between the sum of stain areas on the panel surface and the total panel surface area. This characteristic is called "Area Factor" and this is represented by:

$$
F A=\frac{1}{A T} \sum_{1}^{k} A_{n}
$$

where: $F A$ is the area factor, $A T$ is the panel area, $A n$ is the stain area and $k$ is the number of stains $\left(C_{T}\right)$.

In figure 5, it can be observed that the Area Factor increases proportionally with the stained area of the panel surface, indicating in this way a lower quality.

\subsection{Intensity}

This characteristic indicates the intensity level (gray scale, $0-255$ ) of the stains. This property provides information of the stain type on the panel surface. For example, the humidity stain will have a different intensity than an oil stain. To represent this factor, the stain intensity average value and the stain-free panel intensity average are considered.

This characteristic will be called "Intensity Factor" and is represented by:

$$
F I=\frac{X I-X I_{m}}{X I}
$$




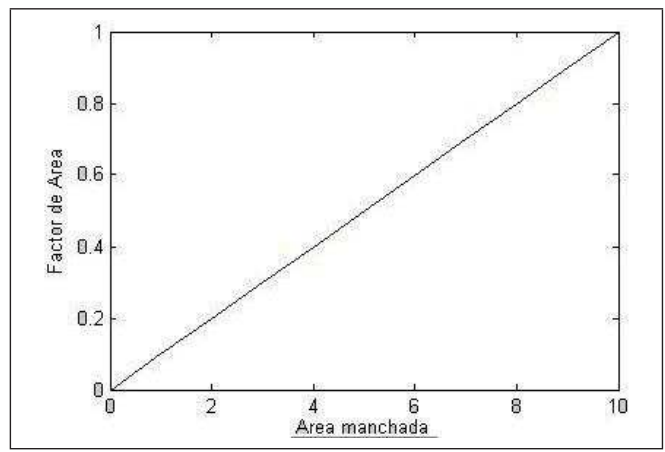

Figure 5: Area Factor v/s Stained Area

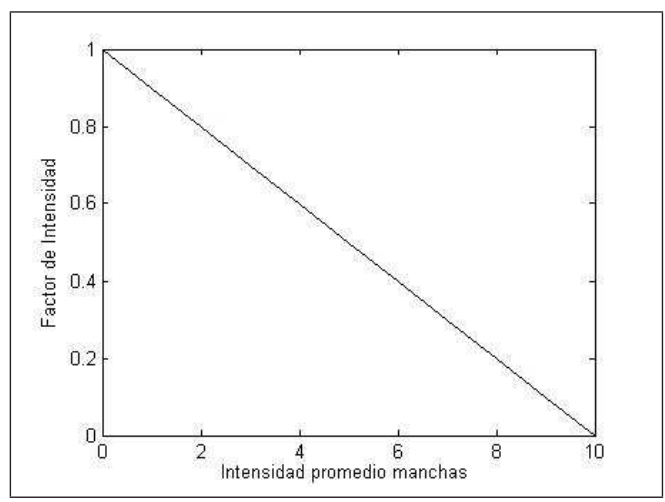

Figure 6: Intensity Factor v/s Average Stain Intensity

where: $X I$ is the stain-free panel intensity average and $X I_{m}$ is the stain intensity average.

Consequently, like can be appreciated in figure 6, the Intensity Factor will increase when stain intensity average value decrease, indicating in this a lower quality.

\subsection{Distribution}

This characteristic determines the spatial location of the stains on the surface. This shows if the stains are grouped in only one panel sector or rather are distributed throughout its surface.

The position of the objects is established through Cartesian coordinates. The method uses the average of the Euclidean distances to the objects geometric centers. The factor is defined by the ratio between the distances average and a reference value. The reference value is the distance from the diagonal to the sample. Expressed in percentage:

$$
F D(\%)=100 *\left[\frac{\mu(D)}{D T}\right]
$$

Where: $\mu(D)$ is the average of Euclidean distances, $D$ is the set of objects, $D T$ is the distance from the diagonal of the sample.

The average of distances $\mu(D)$ depend how distant these object are from the geometric center. The farther they are, the greater is the value of $(D)$ and consequently, $F D(\%)$ also be greater, indicating that the object distribution is greater. In figure 7, three examples of distributions are showed.

As an example, table 2 presents numerical values of the factors proposed in this work, in relation to the grading criteria presented in table 1 . The panel format was $1.5 \times 2.3$ meters. 


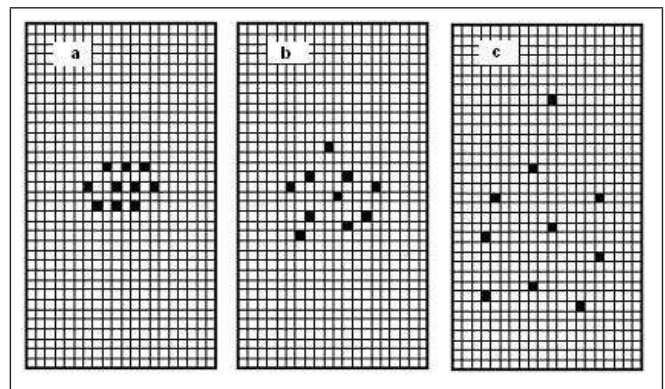

Figure 7: (a) $\mathrm{FD}=6.8 \%$, (b) $\mathrm{FD}=9.05 \%$, (c)FD=17.32\%

\begin{tabular}{ccccccc}
\multicolumn{4}{c}{ MDF } & \multicolumn{3}{c}{ FIBER } \\
\hline QUAL & FC & FA $(\%)$ & FD & FC & FA $(\%)$ & FD $(\%)$ \\
\hline I & $<2$ & $<0,01$ & NE & $<5$ & $<0.07$ & 1,8 \\
\hline II & $-<10$ & NE & - & $<10$ & 7 & \\
\hline III & SEC & SEC & SEC & SEC & SEC & SEC
\end{tabular}

Table 2: Example of Factors (NE: Not Specified, SEC: Without Quality Requirements.)

\section{Comments and conclusions}

This work is focused towards Image Analysis by segmentation of stains present on wood panel surfaces and in the definition of factors to quantify defects properties (Quantity, Area, Intensity and Distribution).

Techniques like thresholding and Transformed Watershed showed goods results to segment stains in gray scales. Thresholding is a useful and simple technique, but when it is used only at one threshold level it is not capable of separating (labeling) the distinct stain intensity values that are below this value.

The Transformed Watershed method offers the capacity of separating regions (stains) in function of the intensity value presented by each defect. However, this technique presents over-segmentation, problem that is solved through the image pre-processing to increase the contrast for the objects of interest and the assignment of local minimums (Markers).

Factors proposed in this work provide the necessary information to define a Stain Quality Index to on -line control of based-wood panels.

\section{Acknowledgements}

This work was supported by the Project INCON-032008 3/R of the Research Secretary, University of Bio-Bio.

\section{References}

[1] Garrido R., Adquisición y Tratamiento Digital de Señales para Visión Artificial, Reporte Final Ingeniería Electrónica, Universidad del Bío-Bio, 2003.

[2] Corma, Corporación Chilena de la Madera, www.corma.cl, 2004.

[3] Mery D., Inspección Visual Automática, Informe Interno, Departamento de Ingeniería en Computación, Universidad de Santiago de Chile, 2003.

[4] Guindos F., Fernandez J., and Peralta M., Visión Artificial con IMtdi, Almería, 2001. 
[5] González R.,Woods R., Tratamiento Digital de Imágenes, Addison-Wesley, 1996.

[6] Olli Silvén and Matti Niskanen, Framework For Industrial Visual Surface Inspections, 6th International Conference on Quality Control by Artificial Vision, Machine Vision Group, University of Oulu, Finland, 2003.

Cristhian Aguilera, Mario Ramos, Gabriel Roa

Universidad del Bío-Bío, Concepción-Chile

Departamento de Ingeniería Eléctrica y Electrónica

Departamento de Ingeniería en Maderas

Laboratorio de Sistemas Automatizados

E-mail: cristhia@ubiobio.cl,mramos@pegasus.dci.ubiobio.cl 